Journal of Mathematical Sciences and Modelling, 4(3)(2021) 110-116
Research Article
Journal of Mathematical Sciences and Modelling

\title{
Intersections of Multicurves on Small Genus Non-Orientable Surfaces
}

\author{
Saadet Öykü Yurttaş ${ }^{1}$ \\ ${ }^{1}$ Dicle University Science Faculty Mathematics Department, 21280, Diyarbakır, Turkey
}

\begin{abstract}
Let $K_{n}(n>1)$ be an $n$-punctured non-orientable surface of genus 2 with 1 boundary component. We give formulae for calculating the geometric intersection number of an arbitrary multicurve with a relaxed multicurve on $K_{n}$ given their generalized Dynnikov coordinates.
\end{abstract}

\begin{tabular}{l} 
Article Info \\
Keywords: Geometric intersection, \\
Generalized Dynnikov coordinates, \\
Multicurves. \\
2010 AMS: 57 N16, $57 M 50$. \\
Received: 6 October 2021 \\
Accepted: 6 December 2021 \\
Available online: 29 December 2021 \\
\hline
\end{tabular}

\section{Introduction}

Throughout the paper we work on a standard model of $K_{n}(n>1)$ as depicted in Figure 1.1. That is, all the punctures and the crosscaps of $K_{n}$ are aligned along the $x$-axis, and that each disk with an asterisk represents a crosscap, which is a graphical representation of a Möbius band (i.e. interior of such disks are removed and antipodal points on the remaining boundary are identified). We say that a simple closed curve in $K_{n}$ is essential if it satisfies the following properties: it is not the core curve of a Möbius band and it doesn't bound an unpunctured disk, a once punctured disk or a Möbius band. A multicurve $\mathscr{L}$ is the homotopy class of a finite union of essential simple closed curves in $K_{n}$. We say that a multicurve is relaxed if each of its connected components intersects the $x$-axis at most twice (see for instance Figure 3.1). We denote by $\mathfrak{L}_{n}$ the set of multicurves in $K_{n}$. Let $\mathscr{L}_{1}, \mathscr{L}_{2} \in \mathfrak{L}_{n}$. Then the geometric intersection number $\imath\left(\mathscr{L}_{1}, \mathscr{L}_{2}\right)$ is defined as

$$
\min \left\{\left|L_{1} \cap L_{2}\right|: L_{1} \in \mathscr{L}_{1}, L_{2} \in \mathscr{L}_{2}\right\}
$$

where $\left|L_{1} \cap L_{2}\right|$ denotes the number of intersections between $L_{1}$ and $L_{2}$.

The fact that the geometric intersection number is preserved under homeomorphisms yields a two step algorithm which works as follows. The first step of the algorithm is a relaxation algorithm finding a homeomorphism sending one of the multicurves to a relaxed one [1,2] and the second provides formulae to calculate the geometric intersection number between an arbitrary multicurve and a relaxed one. This idea is realized in [1] for finitely many times punctured disks coordinatizing multicurves with Dynnikov coordinates and describing the action of the mapping class group (group of isotopy classes of homeomorphisms) using the update rules [3, 1]. In this paper we establish the second step of the aforementioned approach providing formula for each relaxed curve in $K_{n}(n>1)$.

There are various combinatorial descriptions for multicurves on non-orientable surfaces $[4,5]$. In this paper, we shall make use of the generalized Dynnikov coordinate system [5], which provides a one-to-one correspondence between $\mathfrak{L}_{n}$ and a certain subset of $\mathbb{Z}^{2 n+2} \backslash\{0\}$, to generalize the approach in [2] for multicurves in $K_{n}$.

In Section 2 we present necessary terminology and background related with generalized Dynnikov coordinates of multicurves, and introduce some notions which will be important for developing the formulae stated in Section 3.

\section{Generalized Dynnikov Coordinates of Multicurves}

Consider the $\operatorname{arcs} \alpha_{i}(1 \leq i \leq 2 n-2), \beta_{i}(1 \leq i \leq n+1)$ and $\gamma$, and the core curves $c_{1}, c_{2}$ of crosscap 1 and crosscap 2 as shown in Figure 1.1. Given a multicurve $\mathscr{L} \in \mathfrak{L}_{n}$ we can always find a taut representative $L$ of $\mathscr{L}$ that is a representative of $\mathscr{L}$ which intersects each of the arcs and curves minimally. We write $\left(\alpha ; \beta ; \gamma ; c_{1}, c_{2}\right)$ for the set of intersection numbers of $L$ with these arcs and curves. 


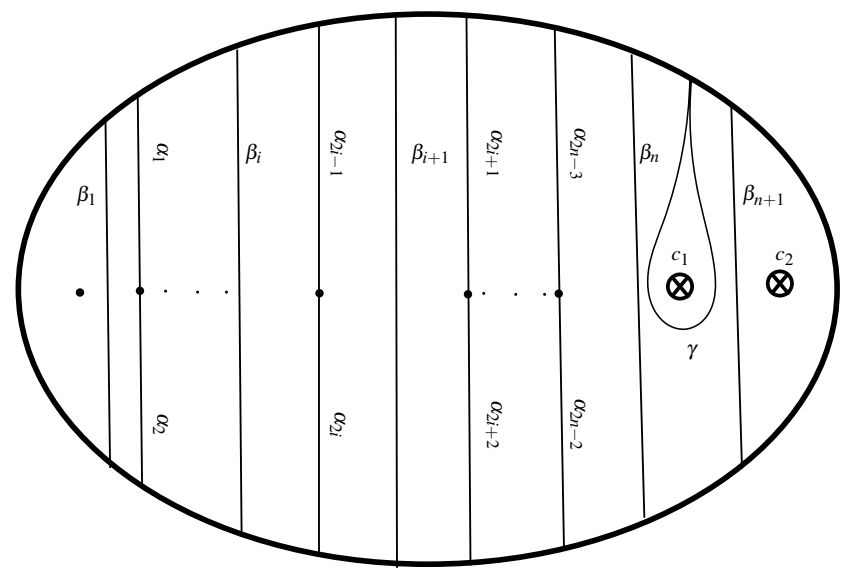

Figure 1.1: The $\operatorname{arcs} \alpha_{i}, \beta_{i}, \gamma$ and curves $c_{1}, c_{2}$ on $K_{n}$

Let $1 \leq i \leq n-1$. Then $S_{i}$ denotes the region which is a subset of $K_{n}$ bounded by $\beta_{i}$ and $\beta_{i+1}$, and contains puncture $i+1$. Note the special interpretation for $S_{0}$ which is bounded by the boundary and $\beta_{1}$. Let $S_{n}$ denote the region bounded by $\beta_{n}$ and $\beta_{n+1}$, and contains crosscap 1 . Similarly, $S_{n+1}$ contains crosscap 2 and bounded by the boundary and $\beta_{n+1}$. We write $S_{i, j}=\bigcup_{k=i}^{j} S_{k}$ for each $i$ and $j$ with $0 \leq i<j \leq n+1$. Then $S_{i, j}$ is the subset of $K_{n}$ bounded by the $\operatorname{arcs} \beta_{i}$ and $\beta_{j+1}$. Note the special interpretation for $S_{0, j}, j \neq n+1$ (resp. $S_{i, n+1}, i \neq 0$ ) which is bounded by the boundary and $\beta_{j+1}$ (resp. $\beta_{i}$ ).

\section{Path components}

Given a taut representative $L \in \mathscr{L} \in \mathfrak{L}_{n}$ we have the following possibilities of a connected component of $L \cap S_{i}$ and $L \cap S_{i, j}$ :

Definition 2.1 (Above components). An above component of $L \cap S_{i}$ has one endpoint on $\beta_{i}$ and the other on $\beta_{i+1}$ passing under puncture $i+1$. Therefore, while it intersects the arc $\alpha_{2 i-1}$ it does not intersect the arc $\alpha_{2 i}$. Similarly, an above component of $L \cap S_{n}$ has one endpoint on $\beta_{n}$ and the other on $\beta_{n+1}$ passing over crosscap 1. Therefore, it intersects the arc $\gamma$ but not the core curve $c_{1}$. An above component of $L \cap S_{i, j}(i \geq 1, j \leq n)$ has one end point on $\beta_{i}$ and the other on $\beta_{j+1}$ and passing entirely over the $x$-axis.

For example, in Figure 2.1 there are 2 above components of $L \cap S_{i}$ and 1 above component of $L \cap S_{i, j}$ for each $2 \leq j \leq n$.

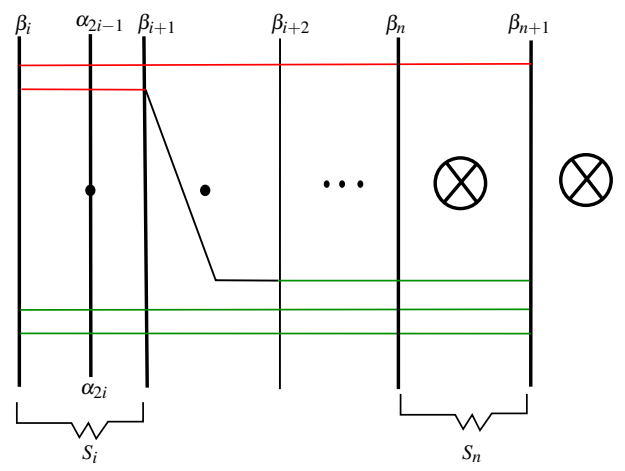

Figure 2.1: Above and below components denoted red and green respectively

Definition 2.2 (Below components). A below component of $L \cap S_{i}$ has one endpoint on $\beta_{i}$ and the other on $\beta_{i+1}$ passing under puncture $i+1$. Therefore, while it intersects the arc $\alpha_{2 i}$ it does not intersect the arc $\alpha_{2 i-1}$. Similarly, a below component of $L \cap S_{n}$ has one endpoint on $\beta_{n}$ and the other on $\beta_{n+1}$ passing under crosscap 1 . Therefore, it neither intersects the arc $\gamma$ nor the core curve $c_{1}$. A below component of $L \cap S_{i, j}(i \geq 1, j \leq n)$ has one end point on $\beta_{i}$ and the other on $\beta_{j+1}$ and passing entirely below the $x$-axis.

For example, in Figure 2.1 there are 2 below components of $L \cap S_{i}$ and $L \cap S_{i, j}$ for each $2 \leq j \leq n$.

Definition 2.3 (Left loop components). A left loop component of $L \cap S_{i}$ intersects each $\alpha_{k}(k=2 i, 2 i-1)$ exactly once and has each of its endpoints on $\beta_{i+1}$. Similarly, a left loop component of $L \cap S_{n}$ intersects $\gamma$ exactly twice having each of its end points on $\beta_{n+1}$. If it intersects the core curve $c_{1}$, we call it a left core loop component, and if it doesn't we call it a left non-core loop component. A left loop component of $L \cap S_{i, j}, j \leq n-1$ intersects the $x$-axis between $\beta_{i}$ and the puncture $i+1$ having each of its end points on $\beta_{j+1}$. A left loop component of $L \cap S_{i, n}$ intersects the $x$-axis between $\beta_{i}$ and the puncture $i+1$ having each of its end points on $\beta_{n+1}$. There are no left loop components of $L \cap S_{i, n+1}$ since there are no above or below components of $L \cap S_{n+1}$. 
Definition 2.4 (Right loop components). A right loop component of $L \cap S_{i}$ intersects each $\alpha_{k}(k=2 i, 2 i-1)$ exactly once and has each of its endpoints on $\beta_{i}$. Similarly, a right loop component of $L \cap S_{n}$ intersects $\gamma$ exactly twice having each of its end points on $\beta_{n}$. If it intersects the core curve $c_{1}$, we call it a right core loop component, and if it doesn't we call it a right non-core loop component. A right loop component of $L \cap S_{i, j}, j \leq n-1$ intersects the $x$-axis between $\beta_{j+1}$ and the puncture $j+1$ having each of its end points on $\beta_{i}$. A right loop component of $L \cap S_{i, n}, i \geq 1$ (respectively $L \cap S_{i, n+1}$ ) intersects the $x$-axis only between crosscap 1 (respectively crosscap 2) and $\beta_{n+1}$ (respectively the boundary) having each of its end points on $\beta_{i}$. There are no right loop components of $L \cap S_{0, j}$ since there are no above or below components of $L \cap S_{0}$.
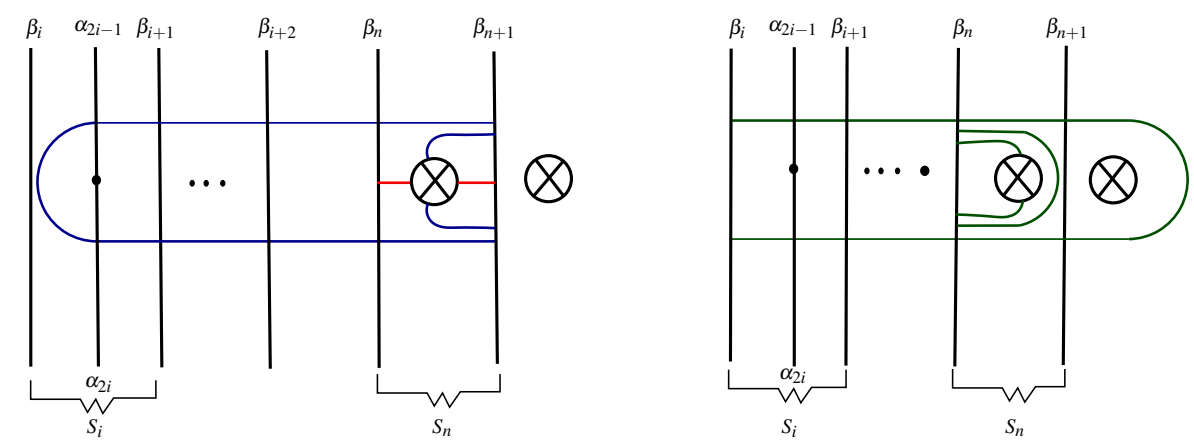

Figure 2.2: Examples for left and right loop components

Definition 2.5 (Straight core components). A straight core component of $L \cap S_{n}$ intersects $c_{1}$ exactly once having one of its endpoints on $\beta_{n}$ and the other on $\beta_{n+1}$. There are no straight core components of $L \cap S_{n+1}$.

Consider for example the left hand side of Figure 2.2. We have 1 left loop component of $L \cap S_{i}, 1$ core loop component and 1 straight core component (depicted red) of $L \cap S_{n}$ and 1 left loop component of $L \cap S_{i, j}$ for each $2 \leq j \leq n$. Similarly, consider the right hand side of Figure 2.2. We have 1 right core and 1 right non-core loop component $L \cap S_{n}$ and 1 right loop component of $L \cap S_{i, n+1}$.

See Lemma 2.3 and Lemma 2.4 in [5] for the proofs of the following lemmas.

Lemma 2.6. Let $1 \leq i \leq n$. There are $\left|b_{i}=\frac{\beta_{i}-\beta_{i+1}}{2}\right|$ loop components of $L \cap S_{i}$. If $b_{i}>0$ the loop components are right and if $b_{i}<0$ they are left. The number of loop components of $L \cap S_{0}$ is given by $\frac{\beta_{1}}{2}$, and the number of right loop components of $L \cap S_{n+1}$ is given by $\frac{\beta_{n+1}}{2}$. We denote by $\lambda_{c_{i}}$ and $\lambda_{i}$ the number of core loop and non-core loop components of $L \cap S_{i}(i=n, n+1)$, and by $\psi$ the number of straight core components of $L \cap S_{n}$.

$$
\begin{aligned}
& \lambda_{1}=\max \left(\left|b_{n}\right|-c_{1}, 0\right) \quad \text { and } \quad \lambda_{c_{1}}=\min \left(\left|b_{n}\right|, c_{1}\right) \\
& \lambda_{2}=\frac{\beta_{n+1}}{2}-c_{2} \\
& \psi=\max \left(c_{1}-\left|b_{n}\right|, 0\right) .
\end{aligned}
$$

Since above and below components of $L \cap S_{i}$ intersect $\alpha_{2 i-1}$ and $\alpha_{2 i}$ respectively; and above and below components of $L \cap S_{n}$ pass above and below crosscap 1 respectively, and that below and above components of $L \cap S_{i, j}$ form the lowest and highest components of each $L \cap S_{i}$ respectively we immediately get Lemma 2.7 .

Lemma 2.7. Denote by $B_{k}$ and $A_{k}$ the number of below and above components of $L \cap S_{k}(1 \leq k \leq n)$. Let $B_{i, j}$ and $A_{i, j}$ denote the number of below and above components of $L \cap S_{i, j}$ respectively. Then, we have

$$
\begin{aligned}
A_{i} & =\alpha_{2 i-1}-\left|b_{i}\right| \quad \text { and } \quad B_{i}=\alpha_{2 i}-\left|b_{i}\right|, \quad \text { for } \quad 1 \leq i \leq n-1 \\
A_{n} & =\frac{\gamma}{2}-\left|b_{n}\right|-\psi \quad \text { and } \quad B_{n}=\max \left(\beta_{n+i}, \beta_{n+i+1}\right)-\left|b_{n}\right|-\frac{\gamma}{2} \\
A_{i, j} & =\min _{i \leq k \leq j} A_{k} \quad \text { and } \quad B_{i, j}=\min _{i \leq k \leq j} B_{k}
\end{aligned}
$$

Notation 1. Let $\lambda_{k}(k=1,2)$ be as given in Lemma 2.6. We write

$$
\lambda_{1}^{+}=\left\{\begin{array}{ll}
\lambda_{1} & \text { if } b_{n}>0 \\
0 & \text { if } b_{n}<0
\end{array} \text { and } \quad \lambda_{i}^{-}= \begin{cases}\lambda_{i} & \text { if } b_{n}<0 \\
0 & \text { if } b_{n}>0\end{cases}\right.
$$

We set $\lambda_{2}^{+}=\lambda_{2}$ since there are only right loop components of $L \cap S_{n+1}$. 


\subsection{The generalized Dynnikov coordinates}

The function $\rho: \mathfrak{L}_{n} \rightarrow \mathbb{Z}^{2 n+2} \backslash\{0\}$ defined by

$$
\rho(\mathscr{L})=\left(a ; b ; t ; c_{1}, c_{2}\right):=\left(a_{1}, \ldots, a_{n-1} ; b_{1}, \ldots, b_{n} ; t ; c_{1}, c_{2}\right)
$$

where

$$
a_{i}=\frac{\alpha_{2 i}-\alpha_{2 i-1}}{2} ; 1 \leq i \leq n-1, \quad b_{i}=\frac{\beta_{i}-\beta_{i+1}}{2} ; 1 \leq i \leq n, \quad t=A_{n}-B_{n},
$$

where $A_{n}$ and $B_{n}$ are as given in Lemma 2.7 is called the generalized Dynnikov coordinate function.

Notation 2. Let $\mathscr{S}=\mathbb{Z}^{2 n+2} \backslash\{0\}$ and $\mathscr{S}_{1}=\left\{\left(a ; b ; t ; c_{1}, c_{2}\right): \in \mathscr{S}:|t|+\psi\right.$ is even $\}$ where $\psi$ is as given in Lemma 2.6.

Next, we give Theorem 2.8 [5] which presents formulae to compute the intersection numbers $\left(\alpha ; \beta ; \gamma ; c_{1}, c_{2}\right)$ from the generalized Dynnikov coordinates $\left(a ; b ; t ; c_{1}, c_{2}\right)$; and hence reconstructs the corresponding multicurve as depicted in Figure 2.3.

Theorem 2.8. Let $\left(a ; b ; t ; c_{1}, c_{2}\right) \in \mathscr{S}_{1}$, and

$$
\begin{aligned}
X & =2 \max _{1 \leq r \leq n-1}\left\{\left|a_{r}\right|+\max \left(b_{r}, 0\right)+\sum_{j=1}^{r-1} b_{j}\right\} \\
Y & =\left\{|t|+2 \max \left(b_{n}, 0\right)+\psi+2 \sum_{j=1}^{n-1} b_{j}\right\} \\
\beta_{i}^{*} & =\max (X, Y)-2 \sum_{j=1}^{i-1} b_{j} \text { and } \quad R=\max \left(0,2 c_{2}-\beta_{n+1}^{*}\right)
\end{aligned}
$$

Then $\left(a ; b ; t ; c_{1} ; c_{2}\right)$ is the generalized Dynnikov coordinate of exactly one element $\mathscr{L} \in \mathfrak{L}_{n}$ with

$$
\begin{aligned}
& \beta_{i}=\beta_{i}^{*}+2 R \\
& \alpha_{i}=\left\{\begin{array}{cl}
(-1)^{i} a_{\lceil i / 2\rceil}+\frac{\beta_{\lceil i / 2\rceil}}{2} & \text { if } b_{\lceil i / 2\rceil} \geq 0, \\
(-1)^{i} a_{\lceil i / 2\rceil}+\frac{\beta_{1+[i / 2\rceil}}{2} & \text { if } b_{\lceil i / 2\rceil} \leq 0,
\end{array}\right. \\
& \gamma=2\left(A_{n}+\left|b_{n}\right|+\psi\right) .
\end{aligned}
$$

Example 2.9. Let $\mathscr{L} \in \mathfrak{L}_{2}$ be a multicurve with generalized Dynnikov coordinates $\rho(\mathscr{L})=(2 ; 1,0 ;-2 ; 2,0)$. Theorem 2.8 gives that $\mathscr{L}$ has intersection numbers $\alpha_{1}=1, \alpha_{2}=5, \beta_{1}=6, \beta_{2}=4, \beta_{3}=4, \gamma=4$. From Lemma 2.6 and Lemma 2.7 we get that $b_{1}=1$ and $b_{2}=0$ that is there is one right loop component of $L \cap S_{1}$ and no loop components of $L \cap S_{2} ; A_{1}=0, B_{1}=4$ that is there are four below components and no above components of $L \cap S_{1}$; and $A_{2}=0, B_{2}=2$ that is there are 2 below components and no above components of $L \cap S_{2}$. Also, $\lambda_{2}=2, \lambda_{c_{2}}=0$ and hence there are no core loop components of $L \cap S_{2}$ and two core loop components of $L \cap S_{2}$. Pasting the pieces of these connected components in each region together uniquely determine the curve as depicted in Figure 2.3.

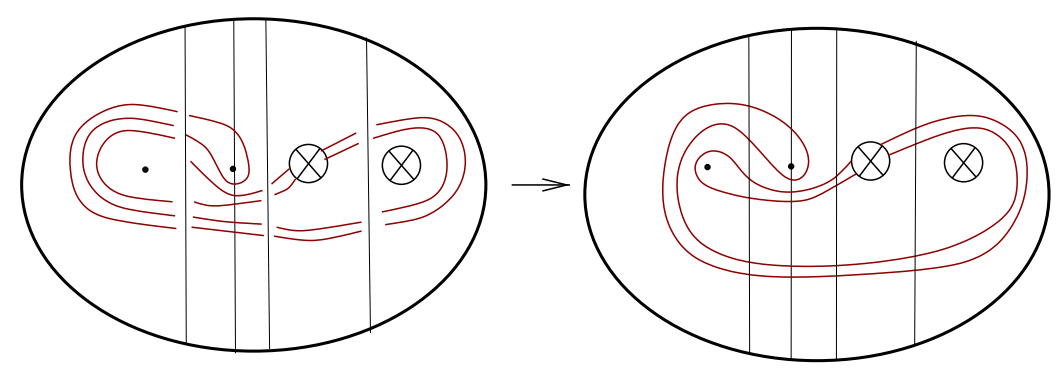

Figure 2.3: Gluing components of $L \cap S_{i}$ together determines $\mathscr{L}$ uniquely up to homotopy

\section{Geometric intersection of multicurves with relaxed curves}

Definition 3.1 (Relaxed curves). A relaxed curve in $K_{n}$ is the homotopy class of an essential simple closed curve in $K_{n}$ which intersects the $x$-axis at most twice, and is represented by one of the following curves:

- $\mathscr{C}_{i, j}$ is contained in the region $S_{i, j}$. It has $\rho\left(\mathscr{C}_{i, j}\right)=(0 ; b ; 0 ; 0) \in \mathscr{S}_{1}$ such that if $0<i<j<n+1, b_{i}=-1$ and $b_{j}=1$. If $i=0$ each $b_{k}=0$ except for $b_{j}=1$, and if $j=n+1$, each $b_{k}=0$ except for $b_{i}=-1$.

- $\mathscr{D}$ is contained in the region $S_{n, n+1}$. It has $\rho(\mathscr{D})=(0 ; b ; 0 ; c) \in \mathscr{S}_{1}$ such that $b_{j}=0(1 \leq j \leq n-1)$ and $b_{n}=-1, b_{n+1}=1$ and $c_{1}=c_{2}=1$.

Notation 3. For convenience we shall denote by $\mathscr{C}$ the homotopy class of the relaxed curve bounding both crosscap 1 and crosscap 2. 


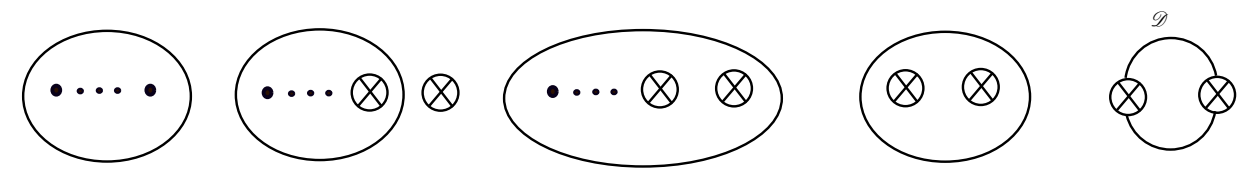

Figure 3.1: Some relaxed curves $\mathscr{C}_{i, j}$ and $\mathscr{D}$ on $K_{n}$

Note that different values for indices $i$ and $j$ give different topological types of curves. Some examples for relaxed curves in $K_{n}$ are illustrated in Figure 3.1. A multicurve $\mathscr{L} \in \mathfrak{L}_{n}$ is relaxed if each of its components is relaxed.

Notation 4. Let $\lambda_{j}^{+}(j=n, n+1)$ and $\lambda_{j}^{-}(j=n)$ be as given in Notation 1. For the sake of brevity we shall write $b_{j}=\lambda_{j}$ for $1 \leq j \leq n$ (this is always possible since there are no core loops about puncture $j$ ).

Lemma 3.2. Let $1 \leq i<j \leq n$. There are $R$ right and L left loop components of $L \cap S_{i, j}$ respectively given by

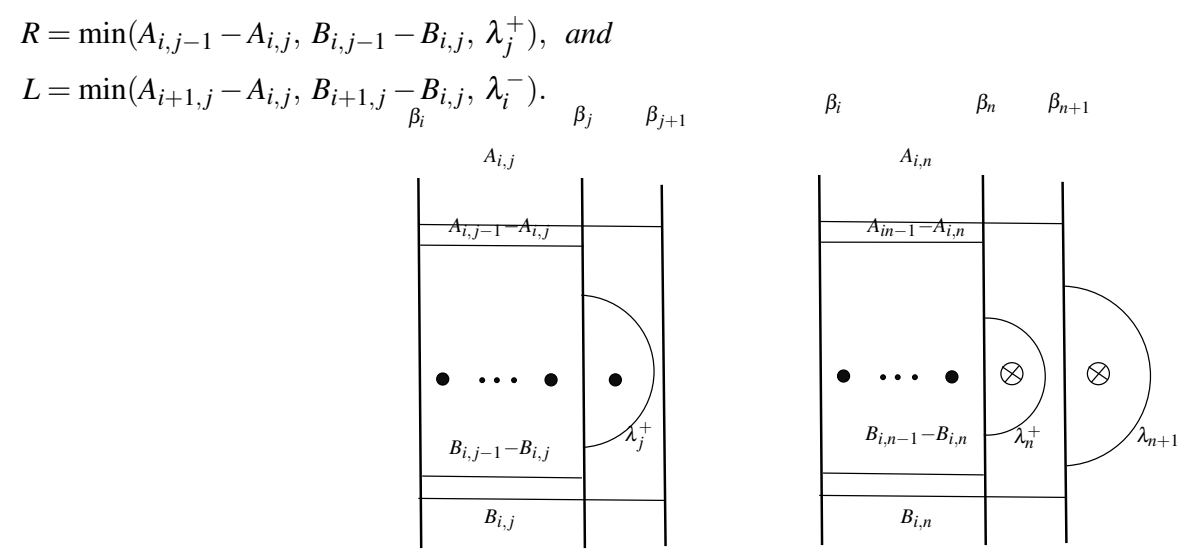

Figure 3.2: Calculation of right loop components of $L \cap S_{i, j}$

Proof. Consider the above components of $S_{i, j-1}$ which are not contained in above components of $L \cap S_{i, j}$. Number of such components is given by $A_{i, j-1}-A_{i, j}$. Similarly, number of below components of $S_{i, j-1}$ which are not contained in below components of $L \cap S_{i, j}$ is given by $B_{i, j-1}-B_{i, j}$. Since there are $\lambda_{j}^{+}$non-core loop components of $S_{j}(j=n, n+1)$ it is immediate from Figure 3.2 that $R$ is the minimum of these three numbers. Number of left loop components of $L \cap S_{i, j}$ is calculated similarly.

Theorem 3.3 (Intersections with $\left.\mathscr{C}_{i, j}\right)$. Let $\mathscr{L} \in \mathscr{L}_{n}$ be a multicurve with $\rho(\mathscr{L})=\left(a ; b ; t ; c_{1}, c_{2}\right) \in \mathscr{S}_{1}$. Let $0 \leq i<j \leq n$ with $(i, j) \neq$ $(0, n+1)$. Then the geometric intersection number $\mathrm{\imath}\left(\mathscr{L}, \mathscr{C}_{i, j}\right)$ is given by

$$
\imath\left(\mathscr{L}, \mathscr{C}_{i, j}\right)=\beta_{i}+\beta_{j+1}-2\left(R+L+A_{i, j}+B_{i, j}\right) .
$$

Proof. Let $\gamma_{i, j}$ be a taut representative of the relaxed curve $\mathscr{C}_{i, j}$, and let $L$ be a taut representative of $\mathscr{L}$ with respect to each arc $\alpha_{i}, \beta_{i}, \gamma$, each curve $c_{i}$, and to $\gamma_{i, j}$. With the set up in Section 2 the proof is identical to that of Lemma 7 in [1] which is based on computing explicitly the number of connected components of $L \cap S_{i, j}$ which are disjoint from $\gamma_{i, j}$. We first note that the number of connected components of $L \cap S_{i, j}$ that are not simple closed curves is given by $\frac{\beta_{i}+\beta_{j+1}}{2}$. Each such component either has zero intersection with $\gamma_{i, j}$ or intersects it twice. Those which are disjoint from $\mathscr{C}_{i, j}$ are above, below, left and right loop components of $L \cap S_{i, j}$ (Figure 3.3) number of which are given by $A_{i, j}, B_{i, j}, L$ and $R$ respectively as given above. Therefore, we get

$$
\imath\left(\mathscr{L}, \mathscr{C}_{i, j}\right)=\beta_{i}+\beta_{j+1}-2\left(R+L+A_{i, j}+B_{i, j}\right)
$$

as required.

Theorem 3.4. Let $\mathscr{L} \in \mathscr{L}_{n}$ be a multicurve with $\rho(\mathscr{L})=\left(a ; b ; t ; c_{1}, c_{2}\right) \in \mathscr{S}_{1}$. Let $\mathrm{\imath}(\mathscr{L}, \mathscr{C})$ and $\boldsymbol{\imath}(\mathscr{L}, \mathscr{D})$ denote the geometric intersection numbers between $\mathscr{L}$ and the relaxed curves $\mathscr{C}$ and $\mathscr{D}$ respectively. Then,

$$
\imath(\mathscr{L}, \mathscr{D})= \begin{cases}\imath(\mathscr{L}, \mathscr{C}) & ; c_{1}=c_{2}=0 \\ \left|c_{1}-c_{2}\right| & ; \text { otherwise }\end{cases}
$$

Proof. There are two cases: Either $c_{1}=c_{2}=0$ or $c_{i} \neq 0$ for some $k \in\{1,2\}$. The former case is immediate from Figure 3.4(a). For the latter case assume without loss of generality that $c_{1} \geq c_{2}$. Then any curve intersecting $c_{1}$ must intersect $c_{2}$ or $\mathscr{D}$ as illustrated in Figure 3.4(b) and Figure 3.4(c). That is, $c_{1}=\mathscr{D}+c_{2}$ as required.

Example 3.5. Let $\mathscr{L} \in \mathfrak{L}_{2}$ be a multicurve with $\rho(\mathscr{L})=(-1 ; 1,0 ; 1 ; 1,1)$ (Figure 3.5). By Theorem 2.8, $\mathscr{L}$ has intersection numbers $\left(\alpha_{1}, \alpha_{2} ; \beta_{1}, \beta_{2}, \beta_{3} ; \gamma_{1} ; c_{1}, c_{2}\right)=(3,1 ; 4,2,2 ; 4 ; 1,1)$. Since $c_{1}=c_{2}=0$, we get from Theorem 3.4 that $\imath(\mathscr{L}, \mathscr{D})=\left|c_{1}-c_{2}\right|=0$. 

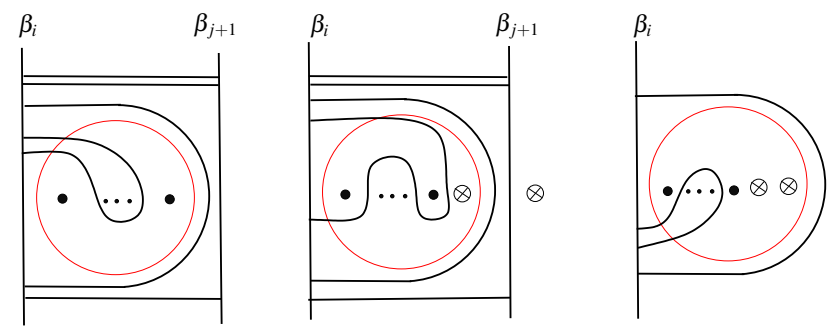

Figure 3.3: Connected components of $L \cap S_{i, j}$ that are disjoint from $\mathscr{C}_{i, j}$

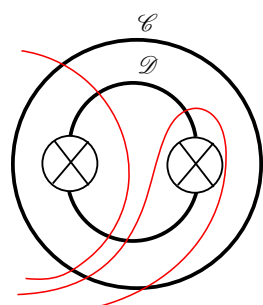

(a)

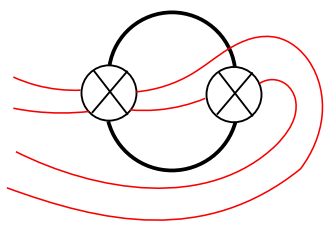

(b)

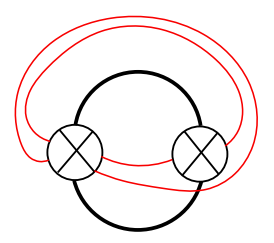

(c)

Figure 3.4: Proof for $\mathscr{D}$

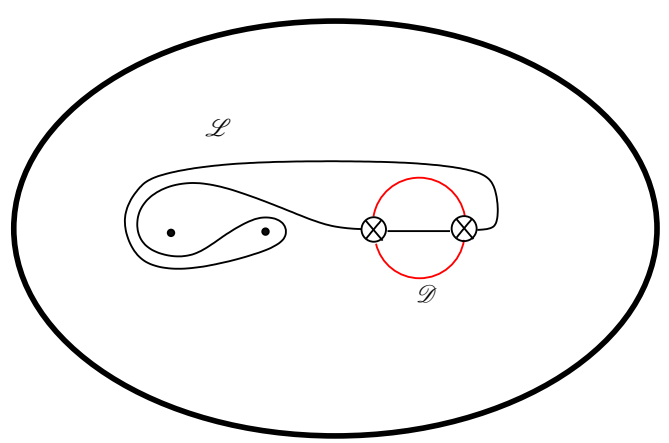

Figure 3.5: $\imath\left(\mathscr{L}, \mathscr{L}_{2}\right)=0$

\section{Conclusion}

The results stated in Theorem 3.3 and Theorem 3.4 are obtained only for genus 2 non-orientable surfaces in this paper. We note that the formulae for relaxed curves which have zero intersection with the crosscaps can be generalized to a higher genus non-orientable surface $N$ immediately using the similar techniques given in Theorem 3.3. Similarly, the formula for $\mathscr{D}$ can be used for the two sided curves $\mathscr{F}_{i, i+1}$ on $N$ which intersects crosscap $i$ and crosscap $i+1$ exactly once, and has zero intersection with the diameter of the surface. However, for relaxed curves $\mathscr{F}_{i, j}$ on $N$ which intersects crosscaps $i$ through $j(j>i+1)$ the method given in Theorem 3.4 fails. The main reason the method doesn't work is that if the arcs intersecting $\mathscr{F}_{i, j}$ are complicated, then it is far from straightforward to describe components which are disjoint from $\mathscr{F}_{i, j}$ or to determine a relation between the number of intersections on $\mathscr{F}_{i, j}$, the core curves and the other relaxed curves $\mathscr{C}_{i, j}$.

Question 1. Generalize the geometric intersection formulae between arbitrary curves and relaxed curves for higher genus non-orientable surfaces. In particular, what is the formula for $\mathscr{L} \in \mathfrak{L}_{g, n}$ and the relaxed curves $\mathscr{F}_{i, j}(j>i+1)$ in terms of their generalized Dynnikov coordinates on higher genus surfaces?

\section{Acknowledgements}

The author would like to express her sincere thanks to the editor and the anonymous reviewers for their helpful comments and suggestions. The author would also like to thank Ferihe Atalan for her valuable comments on an earlier version of this paper.

\section{Funding}

This work was supported by TÜBİTAK (project number 3001-117F282).

\section{Availability of data and materials}

Not applicable. 


\section{Competing interests}

The authors declare that they have no competing interests.

\section{References}

[1] T. Hall, S. Ö. Yurttaş, Intersections of multicurves from Dynnikov coordinates, Bull. Aust. Math. Soc, 98, (2018), 149-158.

[2] S. Ö. Yurttaş, Geometric intersection of curves on punctured disks, J. Math. Soc. Japan, 65(4) (2013), 1153-1168.

[3] I. Dynnikov, On a Yang-Baxter mapping and the Dehornoy ordering, Uspekhi Mat. Nauk, 57(345) (2002), 151-152.

[4] A. Papadopoulos, R.C. Penner, Hyperbolic metrics, measured foliations and pants decompositions for non-orientable surfaces, Asian J. Math, 20 (2016), 157-182.

[5] M. Pamuk, S. Ö. Yurttaş, Integral laminations on non-orientable surfaces, Turkish J. Math, 42 (2018), 69-82. 